\title{
Distal myopathy with rimmed vacuoles: Spectrum of GNE gene mutations in seven Chinese patients
}

\author{
FEIFEI SU* , JING MIAO*, XUEMEI LIU, XIAOJING WEI and XUEFAN YU \\ Department of Neurology, Neuroscience Center, The First Affiliated Hospital of Jilin University, \\ Changchun, Jilin 130021, P.R. China
}

Received December 16, 2017; Accepted June 4, 2018

DOI: $10.3892 /$ etm.2018.6344

\begin{abstract}
Distal myopathy with rimmed vacuoles (DMRV) is a rare, autosomal, recessive inherited disease caused by mutations in the GNE gene. DMRV is an adult-onset disorder characterized by progressive muscle atrophy and weakness, which initially involves the distal muscles with quadriceps sparing. To date, $>150$ GNE mutations have been reported in different populations from around the world. The present study investigated the clinical, pathological and genetic characteristics of seven unrelated DMRV patients from China. Genetic analysis in these patients revealed three novel mutations (c.455_456insC, p.P421L, and p.A287T) and five previously reported mutations (p.D207V, p.C44S, p.G576R, p.A669P, and p.D218G). In addition, the literature on DMRV was reviewed to provide an overview of the disease and broaden the mutational spectrum of the GNE gene in China.
\end{abstract}

\section{Introduction}

Distal myopathy with rimmed vacuoles (DMRV), also known as GNE myopathy, Nonaka myopathy, and hereditary inclusion body myopathy, is an autosomal recessive hereditary distal myopathy (1). DMRV was first described in Iranian-Jewish patients as a vacuolar myopathy affecting the distal muscles of the lower limbs (2). Since then, more and more cases of DMRV have been reported from all over the world, especially after the responsible gene was identified. DMRV is caused by mutations in the UDP- $N$-acetylglucosamine-2-epimerase/ $N$-acetylmannosamine kinase $(G N E)$ gene $(3,4)$. The $G N E$

Correspondence to: Dr Xuefan Yu, Department of Neurology, Neuroscience Center, The First Affiliated Hospital of Jilin University, 71 Xinmin Street, Changchun, Jilin 130021, P.R. China E-mail: xuefan@jlu.edu.cn

*Contributed equally

Key words: genetic analysis, UDP- $N$-acetylglucosamine-2-epim erase $/ N$-acetylmannosamine kinase mutation, quadriceps sparing, muscle atrophy and weakness, muscle biopsy, rimmed vacuoles gene is located on chromosome 9p13-p12. Its major transcript consists of 13 exons, with exons 1 and 13 being non-coding (5). The gene encodes the bifunctional enzyme GNE, which has both epimerase and kinase activity. GNE is a rate-limiting enzyme that catalyzes the initial two steps in the biosynthesis of sialic acid (6). Sialic acid is widely distributed in the tissues and plays an important role in the formation of protein folding. GNE mutations influence and decrease the activity of epimerase and kinase, resulting in the declined level of sialic acid. Abnormal aggregating of the protein, such as including tau protein and amyloid in muscle fibers can be induced by the decrease of intracellular sialic acid. The systems of both ubiquitin proteasome and autophagy lysosome are then activated, which might be the reasons of RVs formation (7).

DMRV manifests as abnormal gait, and weakness and atrophy of the distal muscles of the lower extremities, mainly the tibialis anterior, with little or no involvement of the quadriceps muscles even in the advanced stages of the disease. In fact, quadriceps sparing is a characteristic feature of DMRV (2). The initial presentation is foot drop and abnormal gait resulting from the involvement of the ankle dorsiflexors. DMRV is a progressive disease, and most patients will begin to rely on wheelchairs for mobility about 12 years after the onset of symptoms (8). DMRV does not lead to cardiomyopathy even in the late stages $(9,10)$. Furthermore, no studies have reported respiratory failure in DMRV, except for one study from Japan (11).

Magnetic resonance imaging (MRI) of the muscles is not specific but helps to differentiate DMRV from other conditions such as myositis. Muscle biopsy specimens from patients with DMRV exhibit rimmed vacuoles, protein aggregation, variation in muscle fiber size, and an absence of inflammatory cells $(5,7)$. Serum creatine kinase $(\mathrm{CK})$ is usually within normal limits or only mildly elevated. The identification of GNE mutations in genetic studies is diagnostic. Thus far, more than 150 mutations in the GNE gene have been reported worldwide (12). However, relatively few GNE mutations have been reported from China. The first two cases were reported in 2006, and currently, approximately 90 cases of DMRV along with the associated $G N E$ mutations have been reported in China (13-15).

In this series, we retrospectively analyzed the clinical manifestations, pathological features, and gene mutations in seven unrelated Chinese DMRV patients. We identified eight GNE mutations in these patients, including five known 
mutations (p.D207V, p.C44S, p.G576R, p.A669P, and p.D218G) and three mutations that had not been reported before (c.455_456insC, p.P421L, and p.A287T). We hope that our findings will improve our understanding of the pathogenic mechanisms underlying DMRV and facilitate the diagnosis of DMRV.

\section{Materials and methods}

Clinical data. Seven patients were diagnosed with DMRV in the First Affiliated Hospital of Jilin University, between 2013 and 2016. All of these patients voluntarily joined this study, and provided written informed consent before being enrolled into the study. The experimental protocol was established according to the ethical guidelines of the Declaration of Helsinki, and was approved by the Human Ethics Committee of Jilin University (Changchun, China). All patients underwent detailed clinical evaluation, and the following data were collected from each patient: Family history, age at onset, distribution of muscle weakness, serum CK level, and findings of muscle biopsy, electromyography, muscle MRI, and peripheral blood genetic testing.

Muscle biopsy. Open muscle biopsy was performed in all patients. The muscle specimens were vertically embedded in tragacanth gum, which was applied on a cork. The specimens were then frozen for 20-30 sec, under slight shaking, in isopentane that had been precooled in liquid nitrogen. After this, the specimens were stored at a temperature of $-80^{\circ} \mathrm{C}$. For histological examination, serial frozen sections $(8 \mu \mathrm{m})$ were stained using routine histochemical methods, including hematoxylin-eosin, modified Gomori trichrome, periodic acid Schiff, NADH-tetrazolium, succinate dehydrogenase, cytochrome $\mathrm{C}$ oxidase, and oil red $\mathrm{O}$.

Genetic analysis. GNE mutation analysis was performed in all patients. Genomic DNA was extracted from the peripheral blood by the method of Qiagen FlexiGene DNA kit (Qiagen, AB, Sollentuna, Sweden). Genomic libraries were built after DNA fragmented, and PCR amplification using designed primers (Table I) was applied to screen out a successful built segments of genomic libraries, following by hybrid capture to obtain needed sequencing fragments. The target fragments were enriched by PCR amplification and the products were purified and examined successively after above procedures. Then the NEXTSEQ 500 sequencer (Illumina, Inc., San Diego, CA, USA) was applied to analyze these PCR products to obtain original data. CASAVA software (1.8.2; Illumina, Inc.) was used to convert original data into recognizable base sequences, which were analyzed by biological information analysis system to select mutations that in accordance with clinical features. At last, mutations were further confirmed via Sanger sequencing pedigree validation. NM_001128227 was chosen as a reference sequence in this study (16). In addition, all mutations were further confirmed using the Human Gene Mutation Database, Database of Single Nucleotide Polymorphisms and 1,000 Genomes Project.

Statistical analysis. Data are expressed as the mean \pm standard deviation. Average age and mean duration of the disease were analyzed using Excel (version 2013; Microsoft Corp. Redmond, WA, USA). Descriptive analysis of these data was carried out in results discussion. There were no relevant variance analysis of groups because of the small sample size.

\section{Results}

Clinical features. This study involved two male and five female patients. The patients were aged 26-46 years old, and their disease course ranged from 2-10 years. The average age at onset was 29.8 \pm 4.5 years (range, 23-36 years) and the mean duration of the disease was $6.7 \pm 3.6$ years (range, 2-10 years). Lower-limb weakness was present in four patients, upper-limb weakness in one patient, and both upper- and lower-limb weakness in two patients. None of the patients had a family history of DMRV (Table II). All patients were found to have normal cranial nerve function on nervous system examination. According to the Medical Research Council scale, the muscle strength of the upper and lower extremities was $>3$ in all patients, except for patient \#2, in whom the tibialis anterior muscle strength was 2 . This patient had difficulty in standing and walking, and had the gait associated with foot drop when she first presented at our hospital. The main clinical feature in all patients was weakness and atrophy of the distal limbs, with visible involvement of both the legs and hands (Fig. 1). Four patients had foot drop and duck step. At the time of presentation, all patients were ambulant and did not need to be supported (except for patient \#2). The tendon reflexes were weak or absent, but cognition, cranial nerve function, and coordination were unaffected. No patient complained of paresthesia, and none had cardiac or respiratory problems.

The peak serum CK level was 777 U/1 (normal,40-200 U/1); thus, the CK level was mildly elevated (no more than three times the normal level). Electromyography of the upper and lower limb muscles showed myopathic changes. Three patients (\#2, \#4, and \#7) underwent muscle MRI. Bilateral MRI examination of the calf muscles (Fig. 2) showed obvious involvement of the tibialis anterior, tibialis posterior, extensor digitorum longus, peroneus longus, peroneus brevis, and gastrocnemius. In patient \#4, the muscles in the medial and posterior compartments of the thighs were mainly involved. The main changes observed on muscle MRI were muscle atrophy and steatosis. No obvious inflammatory exudation was observed, and the quadriceps femoris muscles were not involved.

Pathological features. Hematoxylin and eosin staining of muscle biopsy specimens showed rimmed vacuoles, fiber size variation, and basophilic granule deposition around the vacuoles (Fig. 3, picture A, B-1, C-G). Small empty spaces surrounded by tiny red granules were observed in the cytoplasm of muscle fibers on modified Gomori trichrome staining (Fig. 3, picture Bb). Inflammatory cell infiltration was not seen, but degeneration and necrosis of the muscle fibers were found. Internal nuclei were discovered in patients \#3, \#4, and \#7. Among all the patients, patient \#2 (Fig. 3, picture $\mathrm{Ba}$ and $\mathrm{Bb}$ ) had the most atrophic muscle fibers, which is consistent with the severe muscle weakness observed on clinical examination in this patient. Periodic acid Schiff, NADH-tetrazolium, succinate dehydrogenase, cytochrome $\mathrm{C}$ oxidase, and oil red $\mathrm{O}$ staining showed no obvious abnormalities. 
Table I. Primer sequences for polymerase chain reaction.

Primer sequence $\left(5^{\prime}-3^{\prime}\right)$

\begin{tabular}{lll}
\cline { 2 - 3 } Exon no. & \multicolumn{1}{c}{ Forward } & \multicolumn{1}{c}{ Reverse } \\
\hline 3 & CATAAGTGGAGGTGCAAAAAAAGAT & ACATAAAAACTAAGCAGCAGAACAG \\
4 & TTGCAACTCGGAGGTTCGTC & ACTGTGCACGGCAGGAAGAT \\
7 & TGCCCGACCATCTTTCACTT & GCACCCTGTGACCACTGACA \\
10 & TATTTCCTTGCAGTCCTTGGTAAC & ATGTGGCTCACCTTCAGGCTCTAG \\
12 & CAAAGGCTTTAGGGGCAGTG & GACACTGCAAAGCACCTGTC
\end{tabular}

A

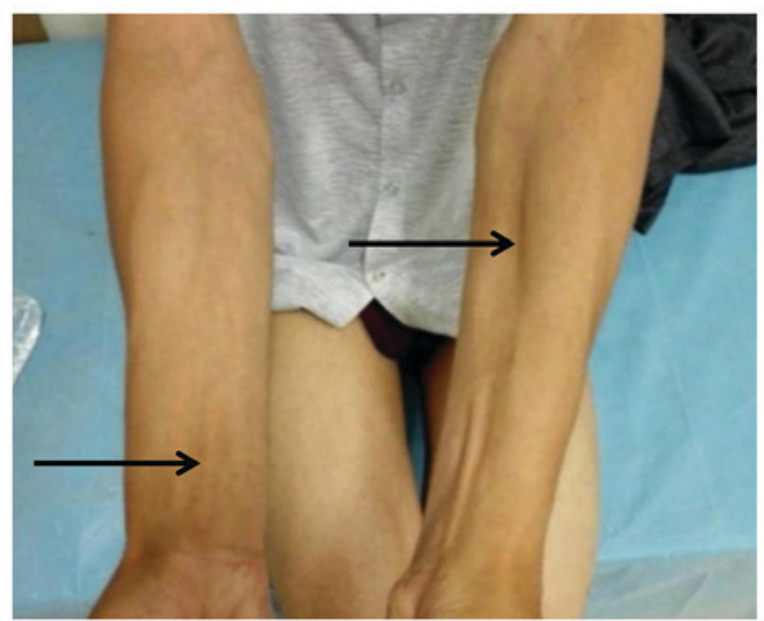

B

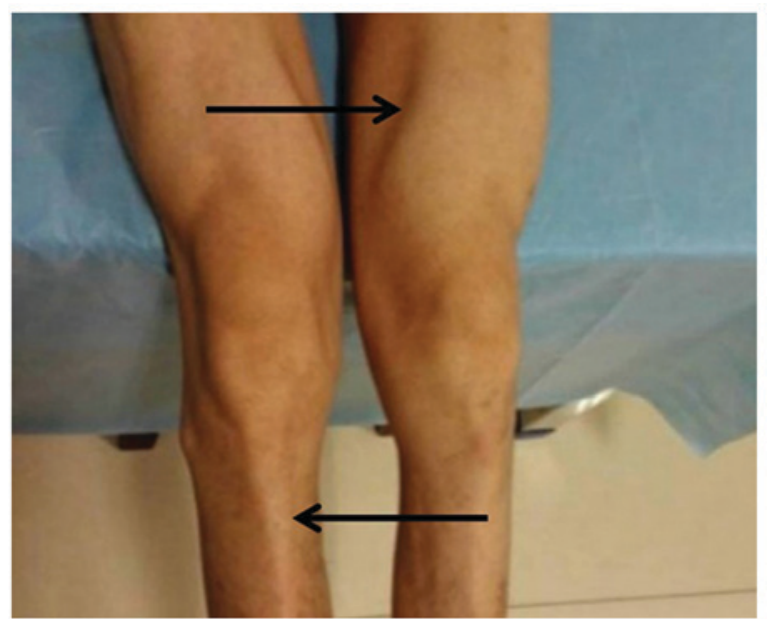

Figure 1. Muscle atrophy in both (A) forearms and (B) calves in patient \#4. The black arrows indicate atrophic muscles.

Genetic features. The GNE genes of the seven patients were analyzed, and the results are shown in Table III. A total of eight different mutations, including one frameshift mutation and seven missense mutations, were detected. Six mutations sites spanned the epimerase region, while two sites were located in the kinase domain. Of the eight mutations, five were known mutations, namely, p.D207V, p.C44S, p.G576R, p.A669P, and p.D218G, while three had never been reported before, namely, c.455_456insC, p.P421L, and p.A287T. Patients \#2, \#4, and \#5 had the same mutation in exon $4(\mathrm{c} .620 \mathrm{~A}>\mathrm{T})$, which resulted in a p.D207V amino acid change, and patients \#3 and \#6 had an identical mutation in exon 3 (c.131G $>$ C), which led to the p.C44S amino acid transformation. Patient \#1 had a frameshift mutation, c.455_456insC, which resulted in the early termination of the codon coding for the amino acid. Patient \#7 had two different mutations in exons 7 and 4 in the epimerase region, resulting in p.P421L and p.A287T amino acid changes.

All of our patients began to have symptoms such as progressive distal and proximal muscle weakness in adulthood, and none of the patients had a family history of DMRV. These clinical features are consistent with previous reports (5). Muscle atrophy was present in five patients; the observation of rimmed vacuoles on muscle biopsy and the identification of GNE mutations on gene analysis confirmed the diagnosis in these patients.

There is no definite connection between phenotype and genotype in DMRV, but it has been reported that a homozygous or heterozygous p.V572L mutation could cause early pathological changes and more obvious clinical symptoms (17). The p.V572L/p.D207V heterozygous mutation has been associated with mild symptoms, while the homozygous p.D207V mutation causes few or even no clinical symptoms. In our sample, the p.V572L mutation was not observed, but one patient had a homozygous p.D207V mutation. This patient had an early onset at the age of 27 years, leading to severe muscle weakness, which is not in accordance with previous reports. We also found two compound heterozygous mutations of p.D207V/p.A669P and p.D207V/p.D218; in both these patients, the muscle strength was $>3$, and symptom onset was relatively late, at the ages of 33 and 30 years, respectively. The patient with the compound heterozygous mutation p.C44S/p.G576R and the one with a homozygous p.C44S mutation had similar muscle strengths and atrophy in the hand muscles, despite having different ages at symptom onset, i.e., 23 and 36 years. The two patients with later onset shared the same missense mutation, p.D207V. Patients \#1 and \#7 had mutations c.455_456insC and p.P421L/p.A287T, respectively, which had never been reported before. Thus, the clinical symptoms in these patients could not be statistically analyzed (12). The age at onset in these patients was 27 and 33 years, respectively (Table II). Patient \#1 had a muscle strength score of $>3$ and a normal CK level, while patient \#7 had a muscle strength score of $>4$ (except in the tibialis anterior) and the highest $\mathrm{CK}$ level in our sample. It is reported that 


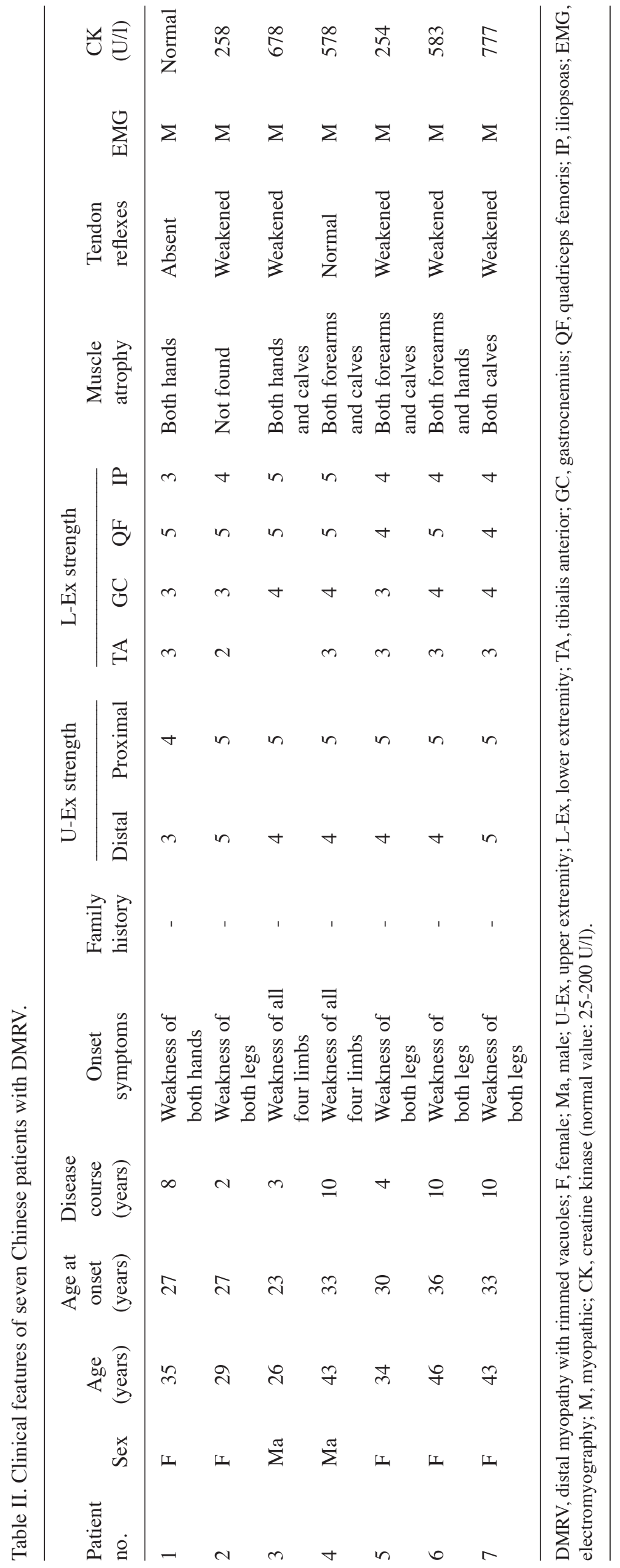


Table III. Genetic analysis results of seven Chinese patients with DMRV.

\begin{tabular}{|c|c|c|c|c|}
\hline Patient no. & Mutation type & GNE mutation & Exon & Domain \\
\hline 1 & Frameshift & c.455_456InsC & 4 & Epimerase \\
\hline 2 & Missense & c.620A>T (p.D207V) & 4 & Epimerase \\
\hline \multirow[t]{2}{*}{3} & Missense & c.131G>C (p.C44S) & 3 & Epimerase \\
\hline & Missense & c.1726G>C (p.G576R) & 10 & Kinase \\
\hline \multirow[t]{2}{*}{4} & Missense & c.620A>T (p.D207V) & 4 & Epimerase \\
\hline & Missense & c.2005G>C (p.A669P) & 12 & Kinase \\
\hline \multirow[t]{2}{*}{5} & Missense & c.620A>T (p.D207V) & 4 & Epimerase \\
\hline & Missense & c.653A>G (p.D218G) & 4 & Epimerase \\
\hline 6 & Missense & c.131G>C (p.C44S) & 3 & Epimerase \\
\hline \multirow[t]{2}{*}{7} & Missense & c.1262C>T (p.P421L) & 7 & Epimerase \\
\hline & Missense & c.859G >A (p.A287T) & 4 & Epimerase \\
\hline
\end{tabular}

DMRV, distal myopathy with rimmed vacuoles; GNE, UDP- $N$-acetylglucosamine-2-epimerase/ $N$-acetylmannosamine kinase.

A

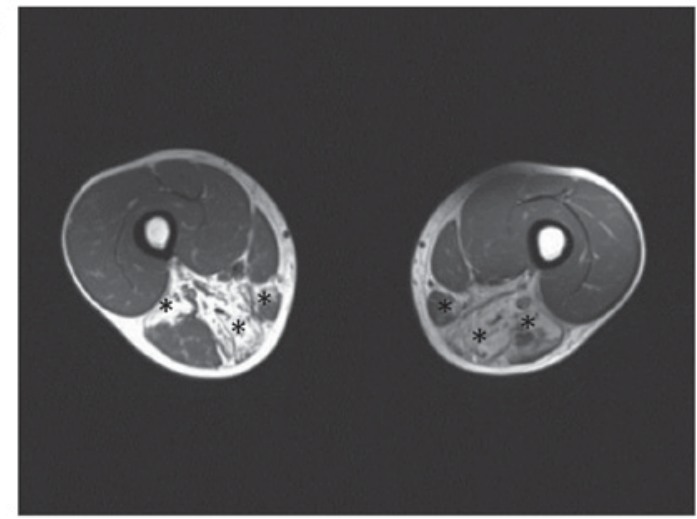

$\mathrm{C}$

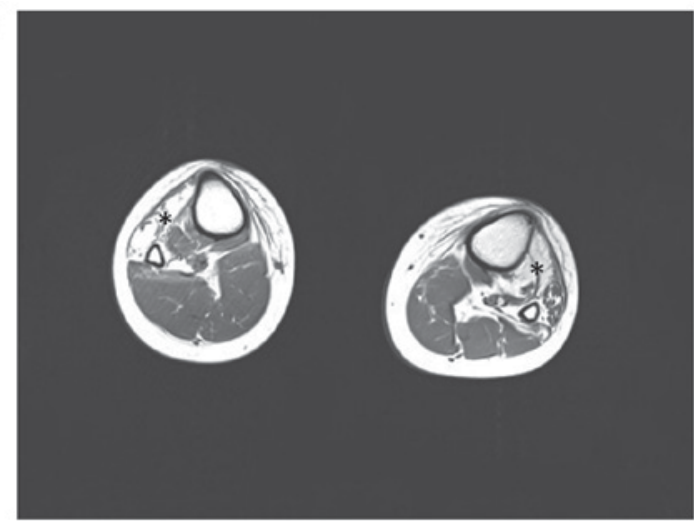

B

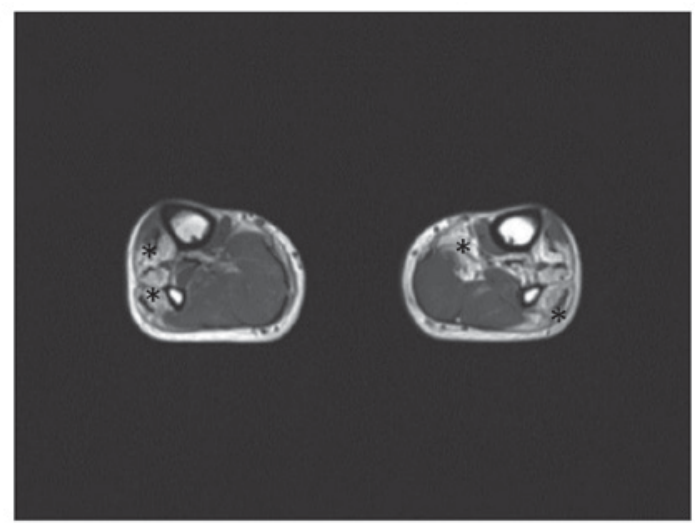

D

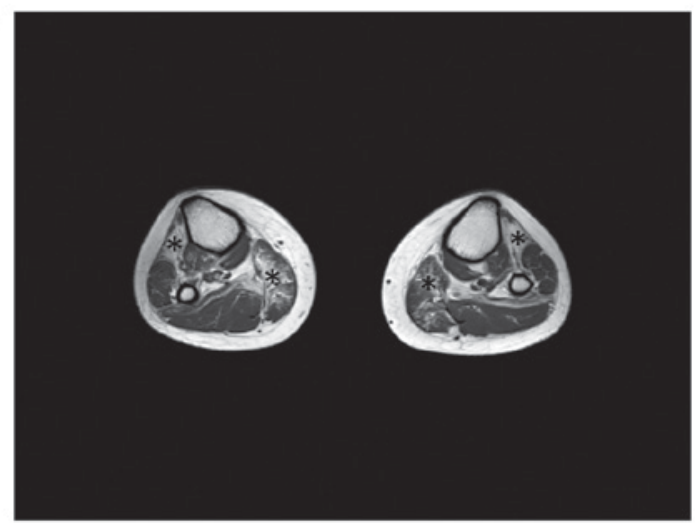

Figure 2. Axial T1-weighted MR images in patient \#4. (A) An MR image was taken in the transverse plane through the thighs shows severe fatty replacement of the muscles in the medial and posterior compartments. (B) An MR image taken in the transverse plane through the calves, shows marked involvement of the tibialis anterior, tibialis posterior, extensor digitorum longus, peroneus longus, peroneus brevis and gastrocnemius. (C) Axial T1-weighted MR images in patient \#2. An MR image taken in the transverse plane through the calves shows marked involvement of the tibialis anterior, tibialis posterior, extensor digitorum longus, peroneus longus, peroneus brevis and gastrocnemius. (D) Axial T1-weighted MR images in patient \#7. An MR image taken in the transverse plane through the calves shows marked fatty replacement of the muscles in the anterolateral and anteromedial areas. The asterisks indicate muscles with fatty replacement. MR, magnetic resonance.

CK results of this disease are ranging from normal or mildly increased level $(8,18)$ to the high level of above $1,000 \mathrm{IU} / \mathrm{l}$ in $23 \%$ of patients (19). DMRV is caused by abnormal proteins depositing in the cytoplasm with the relative integrity of muscle cell membranes. Thus, $\mathrm{CK}$ release is prohibited by the cell membranes and there is no amounts of CK released into the blood to cause a high level of CK (7). Therefore, CK level of DMRV is closely related to the pathogenesis of this disease. Overall, the above results indicate that different mutations lead to different clinical symptoms. However, there is a need 
A
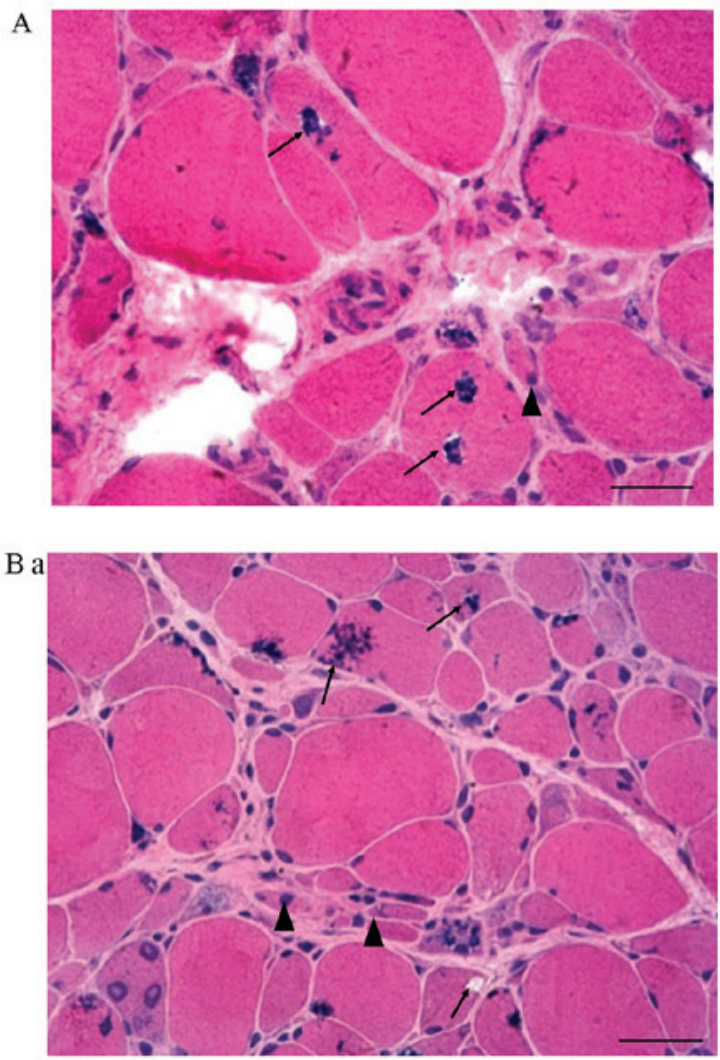

$\mathrm{B}$ b

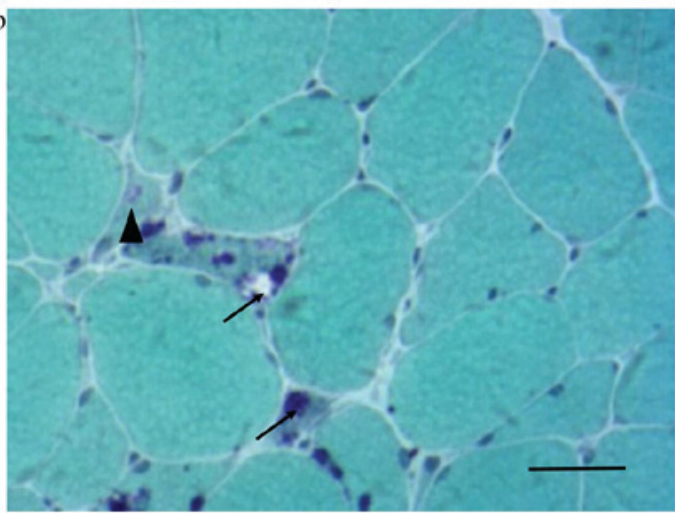

$\mathrm{C}$

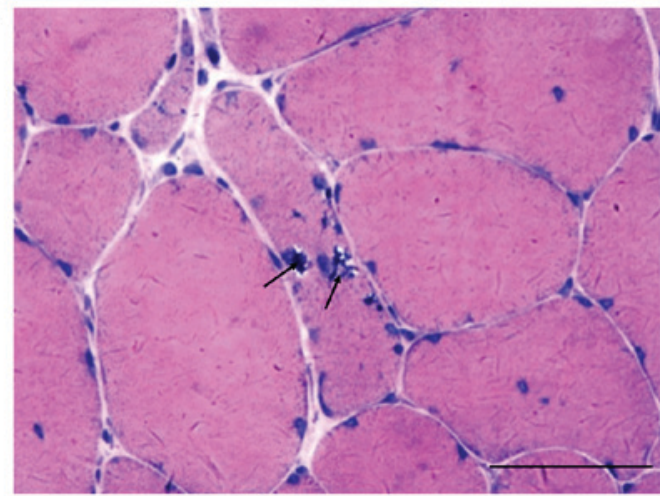

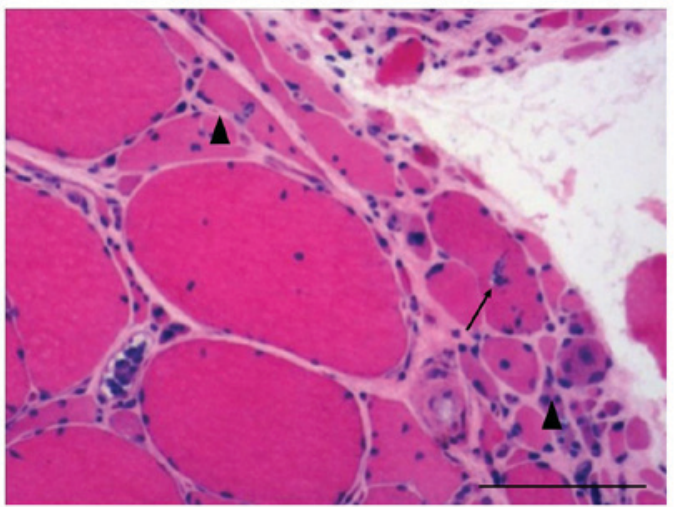

$\mathrm{E}$

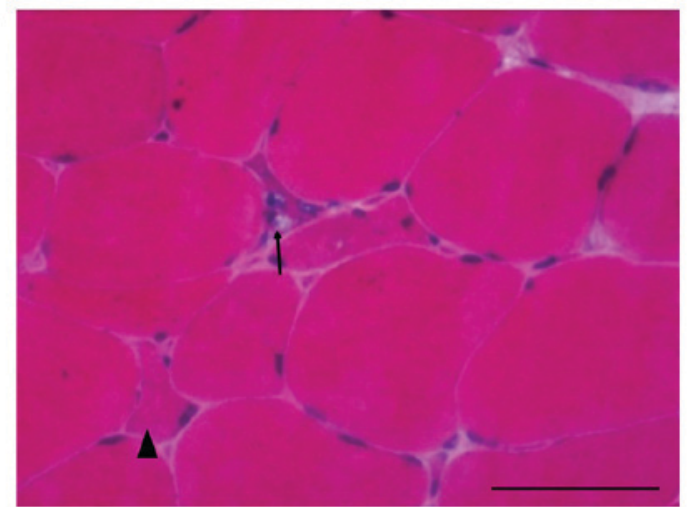

$\mathrm{F}$

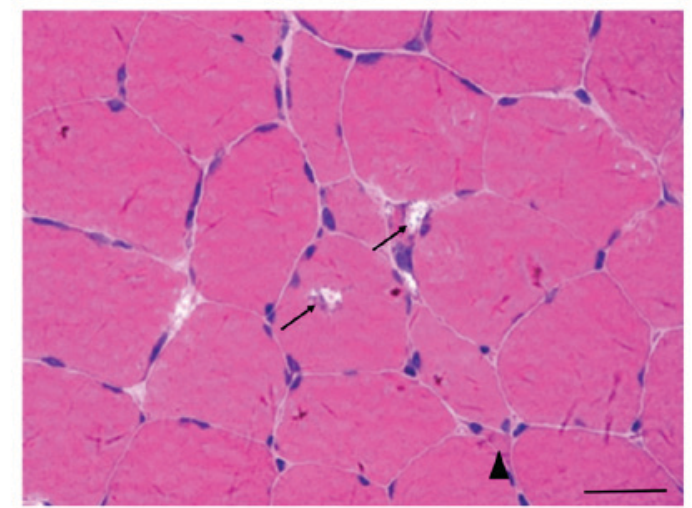

G

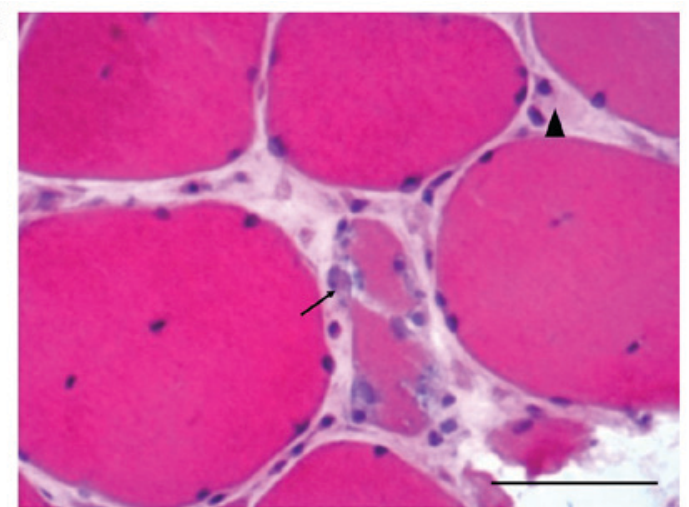

Figure 3. Muscle biopsy examination in all the study patients. (A) Patient \#1, c.455_456insC. Some rimmed vacuoles with basophilic granule deposition and inclusion bodies in angular or round atrophic fibers are seen. Degeneration and necrotic muscle fibers can also be found (hematoxylin and eosin staining; magnification, x200). (Ba) Patient \#2, p.D207V. Variation in muscle fiber size is observed. Some necrotic muscle fibers can be seen (hematoxylin and eosin staining; magnification, x200). (Bb) Patient \#2, p.D207V. Small empty spaces surrounded by tiny red granules in the cytoplasm of muscle fibers (modified Gomori trichrome staining; magnification, x200). (C) Patient \#3, p.C44S/p.G576R. Internal nuclei and atrophic fibers are present (hematoxylin and eosin staining; magnification, $\mathrm{x} 400$ ). (D) Patient \#4, p.D207V/p.A669P. Rimmed vacuoles in two angular fibers and internal nuclei are present (hematoxylin and eosin staining; magnification, $\mathrm{x}$ 400). (E) Patient \#5, p.D207V/p.D218G. Several small, angular, atrophic fibers are seen. Rimmed vacuoles can be observed in one angular fiber (hematoxylin and eosin staining; magnification, x200). (F) Patient \#6, p.C44S. Rimmed vacuoles are seen in a round fiber, and internal nuclei are present (hematoxylin and eosin staining; magnification, x200). (G) Patient \#4, p.D207V/p.A669P. Necrotic fibers with rimmed vacuoles are present (hematoxylin and eosin staining; magnification, x400). Inflammatory cell infiltration is not seen in any panel. Scale bars, $100 \mu \mathrm{m}$. The arrowheads indicate atrophic fibers and the arrows indicate rimmed vacuoles. 
enlarge the sample size to draw definitive conclusions about the connection between phenotype and genotype. A 2012 study described the clinical features of DMRV according to the domain affected by the mutation, i.e., whether the mutation was within the UDP-GlcNAc 2-epimerase domain (ED) or the $N$-acetylmannosamine kinase domain (KD) (10). The authors reported that $\mathrm{KD} / \mathrm{KD}$ mutations are associated with a more severe phenotype than ED/KD mutations, and that ED/ED mutations appear to be associated with a disease severity intermediate between $\mathrm{ED} / \mathrm{KD}$ and $\mathrm{KD} / \mathrm{KD}$ mutations (10). In our series, patients \#5 and \#7 had ED/ED mutations, and had weaker quadriceps femoris and iliopsoas muscles than patients \#3 and \#4, who harbored ED/KD mutations. This finding is consistent with the above report. The connection between phenotype and genotype should be investigated in greater detail in future studies.

\section{Discussion}

DMRV has been reported worldwide in populations of diverse ethnicities. GNE mutations have been reported in Persian Jews, people from the Caucasus region, and the Indian, Thai, Japanese, African, South Korean, and Chinese populations $(1,15,17,20-22)$. These populations show much genetic heterogeneity and at least three instances of the founder effect (12). The first described instance of the founder effect refers to the M712T mutation, which is predominantly found in patients of Middle Eastern descent and also occurs in Muslim people of Bedouin and Palestinian descent as well as among Italian and Japanese people (23-25). The second variant consists of mutations that are common among Japanese patients, namely, p.V572L, p.C44S, and p.D207V (1). The last variant is p.I618T, which is found in the Romani population (10). Korean patients show a high rate of p.V572L, followed by p.C44S (22). With the growing popularity of genetic testing, an increasing number of patients have been diagnosed with DMRV in China. On the basis of relevant papers published by Chinese researchers, we conclude that the mutations p.L508S, p.A631V, p.D207V, and p.A524V have a high incidence in China, and the mutation p.L508S has only been found in China $(13,15)$. The mutation p.V572L, which is relatively common among Japanese patients, was not found in our series, and neither was the mutation p.L508S, which has a high incidence in China. The most common mutation in our study was p.D207V, which is the second most common mutation among Japanese DMRV patients $(1,17)$ and is also very common among Chinese patients (15). Thus, our findings are consistent with the high incidence rates of the p.D207V mutation in these two countries. Two of our patients had the p.C44S mutation, which is seldom reported among Chinese patients but is the third and second most common GNE mutation in Japan and South Korea (17,26), with mutation frequencies of 3.5 and $26.2 \%$, respectively. The geographic relationship among the three countries indicates a possible founder effect of this GNE myopathy. However, the incidences are various, indicating the genotype patterns are not completely consistent though some common mutations are shared among them (15). The mutations p.G576R, p.A669P, and p.D218G, which were reported in 2015 and $2012(10,14)$, were also found in three of our patients. All but two mutations in our study were located in the epimerase region, and the majority of the mutations in our series were missense mutations (Table III); these results are in accordance with previous reports (17). The three previously unknown mutations that we found, namely c.455_456insC, p.P421L, and p.A287T might be beneficial to analyze the hot spot mutations in the future though our observation is limited to a small cohort of patients.

There is still a lack of analysis of hot spot mutations in China probably because of the low incidence rate of DMRV and the lack of recognition of this disease. DMRV is usually diagnosed using clinical and pathological features, though genetic analysis is diagnostic. With increasing knowledge about the mechanisms of this disease, a number of therapeutic methods have been developed and applied, such as supplementation with $\mathrm{N}$-acetylmannosamine, sialic acid, and intravenous immunoglobulin (as a source of sialic acid) (7). However, it remains unclear whether metabolic supplementation can correct the defect or modify the course of the disease.

In conclusion, GNE mutations were detected in all seven patients in this series, confirming the diagnosis of DMRV. This research reported five known mutations and three novel GNE mutations. These results show that there still exist unknown mutations in DMRV, which could be used to enrich the GNE gene spectrum of DMRV myopathy in Chinese patients. Most clinical neurologists are not well aware of DMRV because of the low incidence of the disease, whose diagnosis depends on muscle biopsy and genetic testing. With increasing awareness of DMRV among physicians, we believe that more DMRV cases will be reported in the future.

\section{Acknowledgements}

Not applicable.

\section{Funding}

The present study was supported by the National Natural Science Foundation of China (grant no. 81601088) and the Natural Science Foundation of Jilin Province (grant no. 20160520164JH).

\section{Availability of data and materials}

The datasets used and/or analyzed during the current study are available from the corresponding author on reasonable request.

\section{Authors' contributions}

FS and JM contributed equally to this work. FS was a major contributor in writing the manuscript and interpreting the patients' data. JM designed the genetic test experiments, interpreted the experimental results and critically revised the manuscript for important intellectual content. XY assisted with the writing and editing of the manuscript and interpreted the gene analysis. XL collected the patient data. XW helped to collect and process the data. All authors agreed to be accountable for all aspects of the work in ensuring that questions related to the accuracy or integrity of any part of the work are appropriately investigated and resolved. 


\section{Ethics approval and consent to participate}

The present study was approved by the Human Ethics Committee of Jilin University and all patients provided written informed consent prior to their inclusion.

\section{Patient consent for publication}

All patients provided written informed consent for the publication of their associated images.

\section{Competing interests}

The authors declare that they have no competing interests.

\section{References}

1. Nishino I, Noguchi S, Murayama K, Driss A, Sugie K, Oya Y, Nagata T, Chida K, Takahashi T, Takusa Y, et al: Distal myopathy with rimmed vacuoles is allelic to hereditary inclusion body myopathy. Neurology 59: 1689-1693, 2002.

2. Argov $Z$ and Yarom R: 'Rimmed vacuole myopathy' sparing the quadriceps. A unique disorder in Iranian Jews. J Neurol Sci 64: 33-43, 1984 .

3. Kayashima T, Matsuo H, Satoh A, Ohta T, Yoshiura K, Matsumoto N, Nakane Y, Niikawa N and Kishino T: Nonaka myopathy is caused by mutations in the UDP-N-acetylglucosa mine-2-epimerase/N-acetylmannosamine kinase gene (GNE) J Hum Genet 47: 77-79, 2002.

4. Eisenberg I, Avidan N, Potikha T, Hochner H, Chen M, Olender T, Barash M, Shemesh M, Sadeh M, Grabov-Nardini G, et al: The UDP-N-acetylglucosamine 2-epimerase/ $\mathrm{N}$-acetylmannosamine kinase gene is mutated in recessive hereditary inclusion body myopathy. Nat Genet 29: 83-87, 2001.

5. Huizing $M$ and Krasnewich DM: Hereditary inclusion body myopathy: A decade of progress. Biochim Biophys Acta 1792: 881-887, 2009.

6. Singh R and Arya R: GNE myopathy and cell apoptosis: A comparative mutation analysis. Mol Neurobiol 53: 3088-3101, 2016.

7. Nishino I, Carrillo-Carrasco N and Argov Z: GNE myopathy: Current update and future therapy. J Neurol Neurosurg Psychiatry 86: 385-392, 2015.

8. Nonaka I, Noguchi S and Nishino I: Distal myopathy with rimmed vacuoles and hereditary inclusion body myopathy. Curr Neurol Neurosci Rep 5: 61-65, 2005.

9. Mori-Yoshimura M, Oya Y, Yajima H, Yonemoto N, Kobayashi Y, Hayashi YK, Noguchi S, Nishino I and Murata M: GNE myopathy: A prospective natural history study of disease progression. Neuromuscul Disord 24: 380-386, 2014.

10. Mori-Yoshimura M, Monma K, Suzuki N, Aoki M, Kumamoto T, Tanaka K, Tomimitsu H, Nakano S, Sonoo M, Shimizu J, et al: Heterozygous UDP-GlcNAc 2-epimerase and $\mathrm{N}$-acetylmannosamine kinase domain mutations in the GNE gene result in a less severe GNE myopathy phenotype compared to homozygous $\mathrm{N}$-acetylmannosamine kinase domain mutations. J Neurol Sci 318: 100-105, 2012.

11. Mori-Yoshimura M, Oya Y, Hayashi YK, Noguchi S, Nishino I and Murata M: Respiratory dysfunction in patients severely affected by GNE myopathy (distal myopathy with rimmed vacuoles). Neuromuscul Disord 23: 84-88, 2013.
12. Celeste FV, Vilboux T, Ciccone C, de Dios JK, Malicdan MC, Leoyklang P, McKew JC, Gahl WA, Carrillo-Carrasco N and Huizing M: Mutation update for GNE gene variants associated with GNE myopathy. Hum Mutat 35: 915-926, 2014.

13. Lu X, Pu C, Huang X, Liu J and Mao Y: Distal myopathy with rimmed vacuoles: Clinical and muscle morphological characteristics and spectrum of GNE gene mutations in 53 Chinese patients. Neurol Res 33: 1025-1031, 2011.

14. Liu N, Wang ZK, Wang HX, Li Y, Niu ZH and Yu XF: Muscle biopsy and UDP-N-acetylglucosamine 2-epimerase/N-acetylmannosamine kinase gene mutation analysis in two Chinese patients with distal myopathy with rimmed vacuoles. Neuroreport 26: 598-601, 2015.

15. Zhao J, Wang Z, Hong D, Lv H, Zhang W, Chen J and Yuan Y: Mutational spectrum and clinical features in 35 unrelated mainland Chinese patients with GNE myopathy. J Neurol Sci 354: 21-26, 2015.

16. Huizing M, Carrillo-Carrasco N, Malicdan MC, Noguchi S, Gahl WA, Mitrani-Rosenbaum S, Argov Z and Nishino I: GNE myopathy: New name and new mutation nomenclature. Neuromuscul Disord 24: 387-389, 2014.

17. Cho A, Hayashi YK, Monma K, Oya Y, Noguchi S, Nonaka I and Nishino I: Mutation profile of the GNE gene in Japanese patients with distal myopathy with rimmed vacuoles (GNE myopathy). J Neurol Neurosurg Psychiatry 85: 914-917, 2014.

18. Jay CM, Levonyak N, Nemunaitis G, Maples PB and Nemunaitis J: Hereditary inclusion body myopathy (HIBM2). Gene Regul Syst Bio 3: 181-190, 2009.

19. Tomimitsu H, Shimizu J, Ishikawa K, Ohkoshi N, Kanazawa I and Mizusawa H: Distal myopathy with rimmed vacuoles (DMRV): New GNE mutations and splice variant. Neurology 62: 1607-1610, 2004.

20. Sivakumar K and Dalakas MC: The spectrum of familial inclusion body myopathies in 13 families and a description of a quadriceps-sparing phenotype in non-Iranian Jews. Neurology 47: 977-984, 1996.

21. Liewluck T, Pho-Iam T, Limwongse C, Thongnoppakhun W, Boonyapisit K, Raksadawan N, Murayama K, Hayashi YK, Nishino I and Sangruchi T: Mutation analysis of the GNE gene in distal myopathy with rimmed vacuoles (DMRV) patients in Thailand. Muscle Nerve 34: 775-778, 2006.

22. Kim BJ, Ki CS, Kim JW, Sung DH, Choi YC and Kim SH: Mutation analysis of the GNE gene in Korean patients with distal myopathy with rimmed vacuoles. J Hum Genet 51: 137-140, 2006.

23. Broccolini A, Pescatori M, D'Amico A, Sabino A, Silvestri G, Ricci E, Servidei S, Tonali PA and Mirabella M: An Italian family with autosomal recessive inclusion-body myopathy and mutations in the GNE gene. Neurology 59: 1808-1809, 2002.

24. Kalaydjieva L, Lochmüiller H, Tournev I, Baas F, Beres J, Colomer J, Guergueltcheva V, Herrmann R, Karcagi V, King R, et al: 125th ENMC international workshop: Neuromuscular disorders in the Roma (Gypsy) population, 23-25 April 2004, naarden, the Netherlands. Neuromuscul Disord 15: 65-71, 2005.

25. Eisenberg I, Grabov-Nardini G, Hochner H, Korner M, Sadeh M, Bertorini T, Bushby K, Castellan C, Felice K, Mendell J, et al: Mutations spectrum of GNE in hereditary inclusion body myopathy sparing the quadriceps. Hum Mutat 21: 99, 2003.

26. Sim JE, Park HJ, Shin HY, Nam TS, Kim SM and Choi YC: Clinical characteristics and molecular genetic analysis of Korean patients with GNE myopathy. Yonsei Med J 54: 578-582, 2013. 